\title{
COVID-19: How long does it last?
}

\author{
Hassan I. Osman, MBBS a,b,c,d* \\ ${ }^{\mathrm{a}}$ hassan.io@live.co.uk \\ $\mathrm{a}=$ Department of Psychiatry, Napata College, Sq. 10, Building 151, Al-Riyadh, Khartoum, Khartoum, Sudan. \\ $\mathrm{b}=$ Napata Research and Innovation Center (NRIC), Napata College, Sq. 10, Building 151, Al-Riyadh, Khartoum, Khartoum, Sudan \\ $\mathrm{c}=$ Department of Internal Medicine - Endocrinology Unit - Soba University Hospital - Khartoum, Khartoum, Sudan \\ $\mathrm{d}=$ Reviewer, International Journal of Research Publications (IJRP)
}

\begin{abstract}
COVID-19 has taken the world by storm ever since it first appeared in Wuhan, China back in late 2019. In this paper, we observed a phenomenon that has lately manifested in regards to COVID-19 - that being Long-haul COVID, an illness in which COVID-19 symptoms keep manifesting in patients despite the illness having exceeded its expected course. Findings were rather intriguing; the phenomenon has manifested itself in a plethora of manners which are all discussed in the paper. Furthermore, it is important that we note that the phenomenon is poorly studied and is in need of further exploring. We, at Napata Research Innovation Center pledge to set forth and lead, to the best of our capability, the research efforts in regards to COVID-19 and this newly discussed phenomenon.
\end{abstract}

COVID-19; coronavirus; Napata College; long-haul COVID; Post-COVID-19 Syndrome; Long COVID; Post-COVID-19 Syndrome; Post-COVID-19 symptoms; Severe acute respiratory syndrome coronavirus 2

\section{Main text}

\subsection{Introduction}

'And it's extraordinary how many people [with Covid-19] have a postviral syndrome that's very strikingly similar to myalgic encephalomyelitis/chronic fatigue syndrome. They just don't get back to normal energy or normal feeling of good health.'

- $\quad$ Anthony S. Fauci, MD - Head of the National Institute of Allergy and Infectious Diseases (2)

In 2007, Morens and Fauci published a paper (3) in which they discussed the 1918 pandemic. In that paper, they stated that 'The occurrences of 3 influenza pandemics during the 19th century and of another 3 during the 20th century have led some experts to conclude that pandemics occur in cycles and that we are now overdue.' (3)

More recently (2019) Zhu and colleagues reported a rather peculiar 'pneumonia of unknown cause' $(1,4)$, this 
instigated an investigation which led back to Huanan South China Seafood Market in Wuhan, (1). Eventually,WHO referred to this virus as "2019 novel coronavirus", after which the nomenclature "coronavirus disease (COVID-19)" was adapted (1). As of this writing, we know of 6 coronaviruses in total; those being:
1) $\mathrm{HCoV}-229 \mathrm{E}$
2) $\mathrm{HCoV}-\mathrm{OC} 43$
3) SARS-CoV
4) HCoVNL63
5) HCoV-HKU1
6) $\operatorname{MERS}-\operatorname{CoV}(1)$

Prior to discussing Long-haul COVID-19, became increasingly evident that a proportion of patients has been relatively overlooked by the research community; those being asymptomatic COVID-19 patients. Subsequent attempted to assess the clinical and immunological profiles of these patients (5).

As per the study, the profile is as follows (all of the following are direct quotes from the study) (5):

1) 'The median duration of viral shedding in the asymptomatic group was $19 \mathrm{~d}$ '

2) The asymptomatic group had a significantly longer duration of viral shedding than the symptomatic group

3) The virus-specific IgG levels in the asymptomatic group (median S/CO, 3.4; IQR, 1.6-10.7) were significantly lower $(\mathrm{P}=0.005)$ relative to the symptomatic group (median $\mathrm{S} / \mathrm{CO}, 20.5$; IQR, 5.8$38.2)$ in the acute phase.

4) Of asymptomatic individuals, 93.3\% (28/30) and 81.1\% (30/37) had reduction in $\mathrm{IgG}$ and neutralizing antibody levels, respectively, during the early convalescent phase, as compared to $96.8 \%$ $(30 / 31)$ and $62.2 \%$ (23/37) of symptomatic patients. Forty percent of asymptomatic individuals became seronegative and $12.9 \%$ of the symptomatic group became negative for IgG in the early convalescent phase.

5) Asymptomatic individuals exhibited lower levels of 18 pro-and anti-inflammatory cytokines

Furthermore, the authors commented the following in so far as the data is concerned: 'These data suggest that asymptomatic individuals had a weaker immune response to SARS-CoV-2 infection.' (5).

In 2020, Osman and colleagues of Napata College (6) addressed the cytokine storm issue. In which they hypothesized that the use of Tocilizumab (Actemera) would result in a decreased likelihood of the patient developing a cytokine storm (6). Tocilizumab is an IL-6 targeting therapy focused on the treatment of arthritis (7).

\subsection{Material and methods}

To see to it that the review was properly executed, the authors searched 'PubMed' and 'Google Scholar' as well as 'Google' search engine (the results were, of course, filtered to those of academic reliability) using the entirety of the aforementioned keywords. Following this, they filtered the findings (over 20,000 in number) depending on both their relevance to our topic and their accessibility.

These searches were conducted daily between the $3^{\text {rd }}$ of June 2021 and the $18^{\text {th }}$ of September 2021 so as to avoid the analysis of relatively anachronistic data as well as allow for any updates that became manifest during this time period to appear on the present publication.

Following this, the authors read each and every one of the papers referenced below and illustrated their findings in the manner above. 


\subsection{Literature Review}

'Modern medicine has faced its biggest challenge from the smallest of organisms. It is becoming Increasingly apparent that many patients who recovered from the acute phase of the SARS-CoV-2 infection has persistent symptoms.'

- $\quad$ Avindra Nath, MD(8)

According to a paper by researchers from the University of Glasgow and University College London,

'Long Covid as a term gained consistency in just a few weeks. \#LongCovid was first used byElisa Perego,

from Lombardy, to summarize her experience of disease as cyclical, progressive, and multiphasic. She used

\#LongCovid to intervene ontologically in formulations of COVID-19 in peer-reviewed papers - by integrating the 'biphasic' disease pathway from, and pointing to multiple sequelae.'(9)

Of importance that we note that there exists a genuine need for the arrival at an agreement amongst experts as for the nomenclature of the phenomenon (10)

A number of publications (as well as observations by experts) seem to indicate a fundamental flaw in our understanding of COVID-19 and the specificities associated with it. The primary issue of concern being the disease not occurring in an acute manner as previously believed. Referred to as 'Long-haul COVID, LongCOVID, COVID long-haulers, Long-tail COVID etc.' $(8,11,12)$ As per Nath 'It is becoming increasingly apparent that many patients who recovered from the acute phase of the SARS-CoV-2 infection have persistent symptoms.' (8). According to the same paper, these include 'clouding of mentation, sleep disturbances, exercise intolerance and autonomic symptoms'(8). Other complaints that have been, according to Nath, associated with 'long-haul COVID' are low-grade fever and lymphadenopathy(8).

Of importance, according to Nath, 'Most of these patients were in excellent health prior to getting infected with SARS-CoV-2. They all had a myriad of symptoms during the acute phase. However as the fever and respiratory symptoms improved, they are left with persistent systemic symptoms some of which are gradually improving but not all are following that course.'(8)

In neighboring South Africa, Mendelson and colleagues (11) published an article in which they illustrated the issue in an articulate, clear, and concise manner. The following is a quote from that paper:

'Persistence of symptoms or development of new symptoms relating to SARS-CoV-2 infection late in the course of COVID-19 is an increasingly recognised problem facing the globally infected population and its health systems. 'Long-COVID' or 'COVID long-haulers' generally describes those persons with COVID-19 who experience symptoms for $>28$ days after diagnosis, whether laboratory confirmed or clinical. Symptoms are as markedly heterogeneous as seen in acute COVID-19 and may be constant, fluctuate, or appear and be replaced by symptoms relating to other systems with varying frequency. Such multisystem involvement requires a holistic approach to management of long-COVID, and descriptions of cohorts from low- and middle-income countries are eagerly awaited'(11)

As of this writing, it is currently unknown what percentage of those infected with COVID-19 will go on to suffer from 'long-haul COVID'. It seems, however, that 5 symptoms are shared by $\sim 75 \%$ of those suffering from long-haul COVID, those being (in no particular order):

i. Headache,

ii. SOB,

iii. Fatigue,

iv. Hoarse voice, and 


\section{v. Myalgia(11)}

Furthermore, it also seems as if the groups most likely to experience these symptoms are: i) Women, ii) Older people $(60+)$, and iii) Individuals struggling with obesity $(11,13)$

Data from a UK-based study suggests that, even when patients were asymptomatic, biomarkers associated withstress-response and inflammation remained raised in a significant proportion of them 40 days postinfection(12).

It seems as if another side-effect of COVID-19 that should be placed into consideration is POTS (14). According to data from Sweden, this manifested itself following 'initial outpatient care for coronavirus disease-2019 (COVID-19) infection'(14). Osman and colleagues warned of the possibility of cardiac complications associated with COVID-19 in their 2020 paper(1).

Of late( 2021), researchers from a plethora of medical fields of practice joined forces to explore the topic at hand in Mayo Clinic(15). As per their research,

1) The mean age of participants was 45 years

2) Their participants presented at a mean of 93 days following infection

3) Most of these participants were women (68\%)

4) The most common pre-existing conditions were those regarding mental health (depression, and/or anxiety)

5) The $2^{\text {nd }}$ most common pre-existing conditions were those regarding the respiratory system

6) $75 \%$ of their participants had not been in hospice for the condition

7) The most common presenting symptom was fatigue $(80 \%)$

8) The $2^{\text {nd }}$ most common presenting symptoms were regarding the respiratory and neurological systems (both at 59\%)

9) $34 \%$ of the participants had reported 'difficulties with performing basic activities of daily living'

10) Approximately the same percentage $\left(\frac{1}{3}\right)$ of participants 'had returned to unrestricted work duty'

11) For the most part, the participants' presentations necessitated 'physical therapy, occupational therapy, or brain rehabilitation’(15)

According to a paper out of published inElsevier, the 'post-COVID' symptoms reported were 'fatigue, dyspnoea, chest pain, joint pain, palpitations, anosmia and dysgeusia, hair loss, cognitive symptoms (memory and attention deficits) and psychosocial distress (loneliness, anxiety, depression and sleep disorders)'(16). Another quote from the same paper 'In some of the recovered patients, slow recovery is to be expected. Severe COVID-19 necessitates a prolonged intensive care unit (ICU) stay, and symptoms of 'post-ICU syndrome' are not unique to COVID-19. However, a retrospective study by.... individuals who have recovered from mild and moderate COVID-19 are also experiencing post- COVID-19 symptoms, sometimes with disabling features and inability to return to their normal life. Similar persistent symptoms have been described following severe acute respiratory syndrome and Middle Eastern respiratory syndrome illnesses, although for the latter it was described in patients who had severe disease.'(16).

Although it is virtually established that obesity is negatively associated with COVID-19 infection (i.e. obese individuals are at a high risk for COVID-19 mortality), it is not well-established as to what the effects obesity has on 'long-haul COVID'(13). The authors of a US-based study stated the following 'Findings of this study suggest that moderate and severe obesity (BMI $\geq 35 \mathrm{Kg} / \mathrm{m} 2)$ is associated with a greater risk of PASC.' (13). 
Baig published a commentary regarding the nomenclature of this phenomenon in which they suggested the name 'chronic covid syndrome (CCS)' (10). We agree with this name being adopted to refer to the phenomenon and will be adopting it henceforth.

In January of 2021, the first (as far as our research efforts have concluded) publication discussing chronic (6 months) effects of COVID-19 was published (17). The authors of this paper excluded a number of participants, this is how they elaborated on that point:

'Patients who died before follow-up, patients for whom follow-up would be difficult because of psychotic disorders, dementia, or re-admission to hospital, those who were unable to move freely due to concomitant osteoarthropathy or immobile before or after discharge due to diseases such as stroke or pulmonary embolism, those who declined to participate, those who could not be contacted, and those living outside of Wuhan or in nursing or welfare homes were all excluded.' (17)

Despite this being a point that most would 'glance over', we believe it to be one of great importance as it hints to the possibility of the entirety of the aforementioned list being complications of COVID-19.

In this paper(17), the results were as follows:

1) One patient $(0.041 \%)$ was re-admitted for 'respiratory failurecaused by underlying pulmonary fibrosis'.

2) One patient $(0.041 \%)$ had 'an acutepulmonary embolism due to deep venous thrombosis of lower limbs after discharge'

3) 3 patients $(0.122 \%)^{\text {'d }}$ developed ischaemic strokes'

4) $1.3 \%$ of participants passed 'after discharge mainly due to exacerbation of underlying pulmonary, heart, and kidney disease'

5) Fatigue/muscle weakness were reported in $63 \%$ of participants

6) Sleep difficulties were reported in $26 \%$ of participants

7) Anxiety/depression were reported in $23 \%$ of participants

Also as per the paper, the most common comorbidities were Hypertension, Diabetes, and cardiovascular disease (unspecified in the paper)(17). Also of importance is the fact that $68 \%$ of the study's participants had required oxygen therapy during their admission in hospice, and that $4 \%$ necessitated ICU admission during their stay(17).

The study also reported a higher percentage in women participants(17).

Despite reports indicating the possible inclusion of corticosteroids in COVID-19 treatment regimens (17-19), the findings of the aforementioned cohort report indicated corticosteroids being of no value in increasing the pace of lung recovery in these patients (17).

In Sweden, a case report published in 2021 illustrated the clinical presentations of 3 patients following them having contracted COVID-19 by 3 months (14).This has been briefly alluded to earlier in this paper. The following is a further detailed analysis of the paper:

1) The total number of cases reported was 3

2) All of the patients' clinical presentations suggested a diagnosis of Postural Orthostatic Tachycardia Syndrome (POTS)

3) The first case was of a 42-year-old female who, in March of 2020, developed a number of symptoms suggestive of COVID-19. She did not seek medical attention and recovered. In May of the same year, her symptoms re-manifested themselves, this time accompanied by abdominal pain and odynophagia. This time around, she sought medical attention. However, investigations turned 
negative for COVID-19. A few months later, in July, she developed 'debilitating symptoms of profound exhaustion with associated sinus tachycardia'. Serological tests were, according to authors, 'borderline' for COVID-19. In September, 'she was found unable to stand more than 5 min.' as well as manifested a number of symptoms diagnostic of POTS. Of importance, she did not tolerate $\beta$ blockers, but improved following the prescription of Ivabradine (HCN channel blocker).

4) The second case was of a 28-year-old female who, in March of 2020, developed COVID-19 symptoms; her PCR test was positive. This patient, unlike the former, developed, during her primary clinical manifestation, symptoms that necessitated hospitalization; twice. Her laboratory investigations were, for the most part, normal; CT-chest revealed pericardial effusion. She was managed appropriately. According to the authors, 'An active standing test demonstrated HR increase from 75 to 128 beats/min with concomitant slight increase in systolic BP $(10 \mathrm{~mm} \mathrm{Hg})$ and pronounced orthostatic intolerance'. Following this, in September of the same year, the patient was referred to a tertiary care center for 'post-COVID-19 follow-up'. Her serological tests indicated COVID-19 (IgG antibodies were present). Signs of POTS were found and managed. She went on to then develop GI symptoms, itching, etc. She was still ill by the time the report was published.

5) The $3^{\text {rd }}$ case was of a 37-year-old male. Symptoms also started back in May of 2020 . They were indicative of COVID-19; however, his COVID-19 laboratory investigations were negative. He was not hospitalized at first, but then required so twice. POTS was suspected and then confirmed. He was still ill as of the publication.

In summary, the patients are relatively young, contracted COVID-19, and then developed POTS. 2/3 were ill as of the publication (the paper was published in April 2021). The mechanism via which post-COVID-19 POTS develops remains yet unclear. As illustrated above, 2/3 patients tested negative for COVID-19. Furthermore, it is important that we note that only one of the patients required initial hospitalization for COVID-19.

As per the authors, 'Available management protocols for POTS aim at increasing intake of fluids (water) and salt, physical countermaneuvers, and individually adapted aerobic exercise in recumbent position to help correct the physiological abnormalities.'(14)

A quote from Mendelson and colleagues (11) articulates the clinical manifestation of Long-haul COVID 'Fatigue and headache were almost universal in persons with long- COVID'(11).

As per the aforementioned paper out of the UK, 'Even 40-60 days post-viral infection, we observed a significant remaining inflammatory response in all patients. Proteins that were still affected were associated with the anti-inflammatory response and mitochondrial stress. This indicates that biochemical and inflammatory pathways within the body can remain perturbed long after SARS-CoV-2 infections have subsided even in asymptomatic and moderately affected patients.'(12).

So far, it is unclear if obesity can be considered a risk factor for long-haul COVID (referred to by the author as post-acute sequelae of COVID-19 (PASC)) (13), however, it is a genuine topic worth looking into.

A very important note made by Halpin, O'Connor, and Sivan was the following:

'In previous outbreaks of severe acute respiratory syndrome and the Middle East respiratory syndrome, we did not have the opportunity to intervene early and prevent the chronicity of these longer-term problems.Health services around the globe need to invest in specialist multidisciplinary rehabilitation services now to reverse these symptoms before they become established. This can prevent a future tsunami of chronic disability that is likely to drain healthcare resources and adversely affect our economies.'(20) 
Could CFS/ME be officially recognized as a presentation of Long-haul COVID?

Prior to answering the question above, it is of the importance that we first define CFS/ME. This being justified by the fact that, as per a 2008 paper (21), CFS/ME is poorly understood amongst doctors both in $1^{\text {st }}$ and $3^{\text {rd }}$ world countries. According to the paper,

'When given a typical case vignette of CFS, only $30.8 \%$ of Brazilian specialist doctors mentioned chronic fatigue or CFS as a possible diagnosis, a proportion substantially lower than that observed in Western affluent countries. Similarly, only $16.2 \%$ (95\% CI, 15.1-17.4\%) of Brazilian primary care attenders were aware of CFS, in contrast to $55.1 \%$ (95\% CI, 53.1-57.1\%) of their British counterparts'(21)

Given the findings of our review, combined with the findings of Cho and colleagues, we believe it is of the upmost importance that we address the topic, albeit briefly.

First and foremost, it is important that we see to it that the following notion, , breifly articulated by Brurberg and colleagues (22) in the introduction their 2014 paper, is illustrated:

'Chronic fatigue syndrome (CFS) is a serious disorder' (22)

The most characteristic feature of the illness would be post-exrertional fatigue associated with a number of autonomous, cognitive, and immune manifestations. Currently, it is unclear what factors play a causal role in the bringing about of CFS/ME. However, data seems to suggest a peculiar mix of infectionsand immunological dysfunction being primary causes of the diseases' clinical manifestations,most likely, in some way, shape, or form, interacting with geneticand psychosocialcomponents $(22,23,32,24-31)$.

\subsection{Justification}

It is of importance that the 'chronic' manifestations of COVID-19 are properly understood as they have a number of implications and influence a plethora of decisions. These decisions are not only medical in nature (for example, an employer may not accept an employee requesting sick leave a month following their COVID infection).In her beautifully written personal narrative, Wall of California (33) discusses the issues that have made themselves manifest in her battle with CFS/ME; in her own words, 'In these cases [of CFS/ME], overly physicalist approaches to caring for patients are not likely to be helpful, and a clinician's willingness to listen to a patient's experience of illness becomes essential.' (33). We hypothesize a similar issue manifesting itself with long-haul COVID; despite the fact that there exists far greater exposure and information regarding COVID as opposed to CFS/ME.

\subsection{Conclusion}

Despite evidence for long-haul COVID-19 being a phenomenon is manifesting itself as rather powerful, it is, understandably, not yet clear exactly what array of symptoms constitute the diagnosis. Ergo, it is of the upmost importance that we see to it that the topic is further researched so that we may grasp a better understanding of the topic.

\subsection{Recommendation(s)}

1) Seeing to it that further research projects on the topic take place and that they are done in diverse peoples so as to account for the plethora of variables that are part of the human species.

2) Extreme carefulness and observation by physicians when prescribing $\beta$-blockers to patients manifesting POTS-like symptoms as some patients sometimes do not tolerate it (orthostatic intolerance)(14). 


\subsection{Acknowledgement(s)}

The authors would like to thank Napata College's administration for their former and currently ongoing support.

\subsection{Conflict(s) of interest:}

The authors hereby declare no competing interests.

1.9. Abbreviations (in alphabetical order):

BMI $=$ Body Mass Index

$\mathrm{CCS}=$ Chronic COVID Syndrome

$\mathrm{CFS} / \mathrm{ME}=$ Chronic Fatigue Syndrome/Myalgic Encephalomyelitis

COVID-19 = Coronavirus disease 2019

$\mathrm{CT}=$ Computed Tomography

$\mathrm{HCN}=$ Hyperpolarization-activated Cyclic Nucleotide-gated

ICU = Intensive Care Unit

PASC $=$ Post-Acute Sequelae of COVID-19

$\mathrm{PCR}=$ Polymerase Chain Reaction

POTS $=$ Postural Orthostatic Tachycardia Syndrome

$\mathrm{UK}=$ United Kingdom

US $=$ United States (of America)

$\mathrm{WHO}=$ World Health Organization

\subsection{References:}

1. I. Osman H, Elmamoun M, A. Elgamel A. The COVID-19 situation in Sudan. Plus, a possible lifesaving intervention? Int J Res Publ. 2020;61(1):1-11.

2. Topol EJ, Verghese A, Fauci AS. Fauci to Medscape: "We're All In It Together and We're Gonna Get Through It" [Internet]. Medscape. 2021 [cited 2021 Sep 18]. Available from: https://www.medscape.com/viewarticle/933619\#vp_3

3. Morens DM, Fauci AS. The 1918 Influenza Pandemic : Insights for the 21st Century. JID. 2007;195:1018-28.

4. Huang C, Wang Y, Li X, Ren L, Zhao J, Hu Y, et al. Clinical features of patients infected with 2019 novel coronavirus in Wuhan, China. Lancet. 2020;395(10223):497-506.

5. Long QX, Tang XJ, Shi QL, Li Q, Deng HJ, Yuan J, et al. Clinical and immunological assessment of asymptomatic SARS-CoV-2 infections. Nat Med. 2020;26(8):1200-4.

6. I. Osman H, Elmamoun M, A. Elgamel A. The COVID-19 situation in Sudan. Plus, a possible lifesaving intervention? Int J Res Publ. 2020;61(1).

7. Venkiteshwaran A. Tocilizumab. MAbs. 2009;1(5):432-8.

8. $\quad$ Nath A. Long-Haul COVID. Neurology. 2020;95(13):559-60.

9. Callard F, Perego E. How and Why Patients Made Long Covid. Soc Sci Med [Internet]. 2020;113426. Available from: https://doi.org/10.1016/j.socscimed.2020.113426

10. Baig AM. Chronic COVID syndrome: Need for an appropriate medical terminology for long-COVID and COVID long-haulers. J Med Virol. 2021;93(5):2555-6.

11. Mendelson M, Nel J, Chb MB, Blumberg L, Bch MB, Micro MC, et al. IN PRACTICE Long- 
COVID : An evolving problem with an extensive impact. SAMJ. 2021;111(1):10-2.

12. Doykov I, Hällqvist J, Gilmour KC, Grandjean L, Mills K, Heywood WE. ' The long tail of Covid-19

' - The detection of a prolonged inflammatory response after a SARS-CoV-2 infection in asymptomatic and mildly affected patients [ version 2 ; peer review : 2 approved ]. $2021 ; 1-10$.

13. Aminian A, Bena J, Pantalone KM, Burguera B. Association of Obesity with Post-Acute Sequelae of COVID-19 (PASC). Diabetes Obes Metab [Internet]. 2021;19(216):0-2. Available from: http://www.ncbi.nlm.nih.gov/pubmed/34060194

14. Johansson M, Ståhlberg M, Runold M, Nygren-Bonnier M, Nilsson J, Olshansky B, et al. Long-Haul Post - COVID-19 Symptoms Presenting as a Variant of Postural Orthostatic Tachycardia Syndrome. (J Am Coll Cardiol. 2021;3(4):573-80.

15. Vanichkachorn G, Newcomb R, Cowl CT, Murad MH, Breeher L, Miller S, et al. Post-COVID-19 Syndrome (Long Haul Syndrome): Description of a Multidisciplinary Clinic at Mayo Clinic and Characteristics of the Initial Patient Cohort. Mayo Clin Proc [Internet]. 2021;96(7):1782-91. Available from: https://doi.org/10.1016/j.mayocp.2021.04.024

16. Yelin D, Margalit I, Yahav D, Runold M, Bruchfeld J. Long COVID-19-it's not over until? CMI. 2020;27(January):506-8.

17. Huang C, Huang L, Wang Y, Li X, Ren L, Gu X, et al. 6-month consequences of COVID-19 in patients discharged from hospital: a cohort study. Lancet [Internet]. 2021;397(10270):220-32. Available from: http://dx.doi.org/10.1016/S0140-6736(20)32656-8

18. Sterne JAC, Murthy S, Diaz J V., Slutsky AS, Villar J, Angus DC, et al. Association between Administration of Systemic Corticosteroids and Mortality among Critically Ill Patients with COVID19: A Meta-analysis. JAMA - J Am Med Assoc. 2020;324(13):1330-41.

19. The RECOVERY Collaborative Group. Dexamethasone in Hospitalized Patients with Covid-19. N Engl J Med. 2021;384(8):693-704.

20. Halpin S, O’Connor R, Sivan M. Long COVID and chronic COVID syndromes. J Med Virol. 2021;93(3):1242-3.

21. Cho HJ, Menzes PR, Bhugra D, Wessely S. The awareness of chronic fatigue syndrome : A comparative study in Brazil and the United Kingdom. J Psychosom Res. 2008;64:351-5.

22. Brurberg KG, Fønhus MS, Larun L, Flottorp S, Malterud K. Case definitions for chronic fatigue syndrome/myalgic encephalomyelitis (CFS/ME): a systematic review. BMJ. 2014;

23. KOMAROFF AL, GEIGER AM, WORMSELY S. IgG SUBCLASS DEFICIENCIES IN CHRONIC FATIGUE SYNDROME. Lancet. 1988;1288-9.

24. Buchwald D, Wener MH, Pearlman T, Kith P. Markers of inflammation and immune activation in chronic fatigue and chronic fatigue syndrome. J Rheumatol [Internet]. 1997;24(2):372-6. Available from: https://pubmed.ncbi.nlm.nih.gov/9034999/

25. Lorusso L, Mikhaylova S V., Capelli E, Ferrari D, Ngonga GK, Ricevuti G. Immunological aspects of chronic fatigue syndrome. Autoimmun Rev [Internet]. 2009;8(4):287-91. Available from: http://dx.doi.org/10.1016/j.autrev.2008.08.003

26. Wensaas KA, Langeland N, Hanfvevik K, Mørch K, Eide GE, Rortveit G. Irritable bowel syndrome and chronic fatigue 3 years after acute giardiasis: Historic cohort study. Gut. 2012;61(2):214-9.

27. Komaroff AL. Chronic fatigue syndromes: relationship to chronic viral infections. J Virol Methods. 1988;21(1-4):3-10.

28. Fluge $\varnothing$, Bruland O, Risa K, Storstein A, Kristoffersen EK, Sapkota D, et al. Benefit from blymphocyte depletion using the anti-CD20 antibody rituximab in chronic fatigue syndrome. a doubleblind and placebo-controlled study. PLoS One. 2011;6(10).

29. Kerr JR, Petty R, Burke B, Gough J, Fear D, Sinclair LI, et al. Gene expression subtypes in patients with chronic fatigue syndrome/myalgic encephalomyelitis. J Infect Dis. 2008;197(8):1171-84.

30. Salit IE. Precipitating factors for the chronic fatigue syndrome. J Psychiatr Res. 1997;31(1):59-65. 
31. Kato K, Sullivan PF, Evengård B, Pedersen NL. Premorbid predictors of chronic fatigue. Arch Gen Psychiatry. 2006;63(11):1267-72.

32. Knoop H, Prins JB, Moss-Morris R, Bleijenberg G. The central role of cognitive processes in the perpetuation of chronic fatigue syndrome. J Psychosom Res [Internet]. 2010;68(5):489-94. Available from: http://dx.doi.org/10.1016/j.jpsychores.2010.01.022

33. Wall D. AMA Journal of Ethics 2019. AMA J Ethics. 2021;21(1):590-5. 